ESAIM: PROCEEDINGS AND SURVEYS, August 2021, Vol. 71, p. 163-174

Didier Auroux, Jean-Baptiste Caillau, Régis Duvigneau, Abderrahmane Habbal, Olivier Pantz, Luc Pronzato, Ludovic Rifford

\title{
DISCRETE HOTELLING PURE LOCATION GAMES: POTENTIALS AND EQUILIBRIA
}

\author{
TAKUYA IImura ${ }^{1}$ AND PierRe vON MOUChE ${ }^{2}$
}

\begin{abstract}
We study two-player one-dimensional discrete Hotelling pure location games assuming that demand $f(d)$ as a function of distance $d$ is constant or strictly decreasing. We show that this game admits a best-response potential. This result holds in particular for $f(d)=w^{d}$ with $0<w \leq 1$. For this case special attention will be given to the structure of the equilibrium set and a conjecture about the increasingness of best-response correspondences will be made.

Résumé. Nous étudions les jeux de localisation pure Hotelling discrets unidimensionnels à deux joueurs en supposant que la demande $f(d)$ en fonction de la distance $d$ est constante ou strictement décroissante. Nous montrons que ce jeu admet un potentiel de meilleure réponse. Ce résultat vaut notamment pour $f(d)=w^{d}$ avec $0<w \leq 1$. Dans ce cas, une attention particulière sera accordée à la structure de l'ensemble d'équilibre et une conjecture sur la croissance de la correspondance de meilleure réponse sera faite.
\end{abstract}

\section{INTRODUCTION}

In his seminal paper [6], Hotelling presents a location model of two competing retailers. Since then, this model has triggered an increasing flow of research in industrial organization. ${ }^{1}$ The pure location part of that model concerns a game in strategic form with a common real segment as strategy set ("Main Street" [6] ). Normally demand is assumed to be inelastic (i.e. not dependant on the distance). The more general case, allowing also for elastic demand, has been thoroughly studied in $[2,17]$; we further refer to this location game as the $\mathrm{cHg}$ (i.e., continuous Hotelling game).

In the $\mathrm{cHg}$ payoff functions are discontinuous. As far as we know there is no general result in terms of the primitives of the game, like that in [10], that guarantees (Nash) equilibrium existence for the $\mathrm{cHg}$. The proof of equilibrium existence in [2] is "by hand", by determining the equilibrium set. As shown in [8] a deeper reason for the $\mathrm{cHg}$ to have an equilibrium is that this game is a potential game. To be more specific, if the demand is highly elastic to the extent that the Principle of Minimum Differentiation fails, then it has a continuous best-response potential; and if not that elastic it has a continuous quasi-potential. In the present article we deal with the discrete variant of the $\mathrm{cHg}$, which we shall refer to as discrete Hotelling game $(\mathrm{dHg})$. Our main result is that the $\mathrm{dHg}$ is always a best-response potential game, a stronger result compared to $\mathrm{cHg}$. We also scrutinize

\footnotetext{
1 Tokyo Metropolitan University, Tokyo, Japan

2 Wageningen University, Wageningen, The Netherlands

${ }^{1}$ It may be good to note that this model also has its merits in the Hotelling-Downs model in voting theory (see, for instance, [4]). Especially here a discrete setting is asked for, as it may not be appropriate to model the number of voters (which may be "very finite") by a continuum.
}

(C) The authors. Published by EDP Sciences, SMAI 2021

This is an Open Access article distributed under the terms of the Creative Commons Attribution License (https://creativecommons.org/licenses/by/4.0), which permits unrestricted use, distribution, and reproduction in any medium, provided the original work is properly cited. 
the condition for the Principle to hold in a special subclass of $\mathrm{dHg}$ for which demand has the exponential form $f(d)=w^{d}$, since this Principle, which explains the tendency of agglomeration, is at the heart of location theory.

Now let us have a quick look to the literature for discrete Hotelling games. Contrary to the continuous variant, this variant only got a little attention in the literature. As far as we know the first article to deal with such a game was [16]. It deals with two players and considers inelastic as well as elastic demand; however, the game deals with "relative payoffs" instead of the usual ones. Mixed Nash equilibria in case of inelastic demand with three players are studied in [7]. Games on a network (also called "Voronoi games") are studied in [5,14]. A theory for games with a finite number of players and inelastic demand where consumers have strict preference over the possible locations is developed in [12]. To our knowledge, [18] is the first article that theoretically analyses $^{2}$ Nash equilibria of two-player discrete Hotelling games under a setting of inelastic and elastic demand by means of the demand function $f(d)=w^{d}$ where $0<w \leq 1$; so inelastic demand if $w=1$ and elastic demand if $w \neq 1$. In [18] it was proven (again) "by hand" that this game has an equilibrium by determining the equilibrium set. As we mentioned, our main result, i.e. Theorem 4.1, shows that the the dHg is a best-response potential game.

The present article is concerned with two active areas of research in game theory: location games and games having a (pure) Nash equilibrium. Although the former already has wide range of applications, and the latter is being studied in more general frameworks, location games are one of major areas in which one still does not have general results on the existence of equilibria. Our aim is to shed some new light on such a theoretical aspect of location games.

The article is further organized as follows. In Section 2 we formally define the $\mathrm{dHg}$. Section 3 makes some useful observations about equilibria of games with location and player symmetry. In Section 4 , we show among other things that the $\mathrm{dHg}$ is a best-response potential game. Section 5 considers the structure of the equilibrium set for the case $f(d)=w^{d}$; in order to obtain this structure the main result in [18], giving explicit formulas for the equilibrium set, is further studied. Section 6 deals for this case with a conjecture about best-response correspondences. Finally, Section 7 compares results for the dHg with those of the $\mathrm{cHg}$.

\section{Setting}

Let $S$ denote an integer interval $\{0,1, \ldots, m\}$ where $m$ is a positive integer and $f: S \rightarrow \mathbb{R}$ a constant or strictly decreasing positive function.

In this article we understand by a discrete Hotelling game $(\mathrm{dHg})$ a two-player game in strategic form, with player set $\{1,2\}$, common strategy set $S$ and payoff functions $u_{i}: S \times S \rightarrow \mathbb{R}$ given by

$$
u_{i}\left(x_{1}, x_{2}\right):=\sum_{y \in V_{i}\left(x_{1}, x_{2}\right)} f\left(\left|y-x_{i}\right|\right)+\sum_{y \in V_{0}\left(x_{1}, x_{2}\right)} \frac{f\left(\left|y-x_{i}\right|\right)}{2} .
$$

Here $V_{0}\left(x_{1}, x_{2}\right)=\left\{y \in S|| y-x_{1}|=| y-x_{2} \mid\right\}$ and, with $\{i, j\}=\{1,2\}, V_{i}\left(x_{1}, x_{2}\right)=\left\{y \in S|| y-x_{i}|<| y-x_{j} \mid\right\}$. We refer to $f$ as a demand function. The case where $f$ is constant (not constant) also is referred to as inelastic (elastic) demand. Note that the $\mathrm{dHg}$ is a symmetric game.

Short possible real-world interpretation: $S$ represents a space of $m+1$ locations. Each location is occupied by consumers. There are two players, being retailers, who independently and simultaneously choose a location (may be the same). Next, the consumers of each location, say location $x$, will shop at a retailer who is located on a for these consumers best location in the sense that it is a closest one. If this location is unique, say $y$, then the consumers of location $x$ contribute a payoff equal to $f(|y-x|)$ to the retailer at $y$. If there are two locations which are best, then both retailers receive a payoff equal to $f(|y-x|) / 2$.

\footnotetext{
${ }^{2}$ However, [1] already provides simulation results.
} 
Define $L, R: S \rightarrow \mathbb{R}$ and $M: S \times S \rightarrow \mathbb{R}$ by

$$
L(a):=\sum_{d=0}^{a} f(d), \quad R(b):=\sum_{d=0}^{m-b} f(d), \quad M(a, b):=\sum_{d=1}^{\left\lfloor\frac{|a-b|}{2}\right\rfloor} f(d)+\sum_{d=1}^{\left\lfloor\frac{\lfloor a-b \mid-1}{2}\right\rfloor} f(d)
$$

where $\lfloor x\rfloor$ denotes the largest integer not exceeding $x .^{3}$ Using these expressions, the payoff functions can be rewritten as follows:

$$
u_{i}\left(x_{1}, x_{2}\right)= \begin{cases}L\left(x_{i}\right)+\frac{1}{2} M\left(x_{1}, x_{2}\right) & \text { if } x_{i}<x_{j}, \\ R\left(x_{i}\right)+\frac{1}{2} M\left(x_{1}, x_{2}\right) & \text { if } x_{i}>x_{j}, \\ \frac{1}{2}\left(L\left(x_{i}\right)+R\left(x_{i}\right)-f(0)\right) & \text { if } x_{i}=x_{j} .\end{cases}
$$

As there are two players, the game can be represented in a natural way as a $m \times m$ bi-matrix game; rows and columns are numbered from 0 to $m$. A special case, extensively studied in [18] and reconsidered below, is where the demand function $f$ is

$$
f(d)=w^{d}
$$

with $0<w \leq 1$; here we refer to $w$ as distance factor. Here is a visualization in the case $m=7$ and $w=1 / 2$ for the situation of the strategy profile $(2,6)$ :

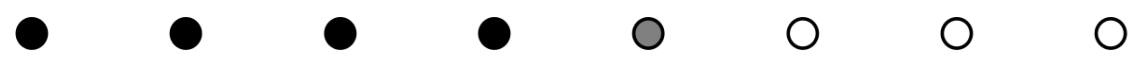

Locations 0, 1, 2, 3 (in black) completely contribute to the payoff of player 1, locations $5,6,7$ (in white) completely to the payoff of player 2 and location 4 (in gray) is shared. The payoffs are $u_{1}(2,6)=\frac{1}{4}+\frac{1}{2}+1+\frac{1}{2}+\frac{1}{8}=$ $\frac{11}{8}$ and $u_{2}(2,6)=\frac{1}{8}+\frac{1}{2}+1+\frac{1}{2}=\frac{17}{8}$. And for $m=3$ and general distance factor $w$ this demand function gives, in bi-matrix terms, for player 1 the payoffs

$$
\left(\begin{array}{cccc}
\frac{1+w+w^{2}+w^{3}}{2} & 1 & 1+\frac{w}{2} & 1+w \\
1+w+w^{2} & \frac{1+2 w+w^{2}}{2} & 1+w & 1+w+\frac{w}{2} \\
1+w+\frac{w}{2} & 1+w & \frac{1+2 w+w^{2}}{2} & 1+w+w^{2} \\
1+w & 1+\frac{w}{2} & 1 & \frac{1+w+w^{2}+w^{3}}{2}
\end{array}\right)
$$

It will be convenient to let

$$
p:=\left\{\begin{array}{l}
m / 2 \text { if } m \text { is even, } \\
(m-1) / 2 \text { if } m \text { is odd. }
\end{array}\right.
$$

Our interest is the set $E$ of Nash equilibria of a $\mathrm{dHg}$.

\section{Games With PlAyer AND LOCATION SyMmetry}

In this section $X=[0, L]$ where $L$ is a positive real number ("continuous case") or $X=\{0,1, \ldots, m\}$ with $m$ a positive integer ("discrete case") and we consider a game in strategic form with two players 1 and 2 , with common strategy set $X$ and with payoff functions $u_{1}, u_{2}: X \times X \rightarrow \mathbb{R}$.

We assume player symmetry, i.e.

$$
u_{2}\left(x_{1}, x_{2}\right)=u_{1}\left(x_{2}, x_{1}\right)\left(x_{1}, x_{2} \in X\right)
$$

and, denoting $L$ and $m$ also by $v$, location symmetry, i.e.

$$
u_{i}\left(x_{1}, x_{2}\right)=u_{i}\left(v-x_{1}, v-x_{2}\right)\left(x_{1}, x_{2} \in X\right) .
$$

\footnotetext{
${ }^{3}$ It is to be understood that the sum in the definition of $M(a, b)$ is zero if the upper bound of the summation is less than unity.
} 
The $\mathrm{cHg}$ (see Section 7 for a formal definition) and the $\mathrm{dHg}$ are examples of such a game. Let $E$ be the Nash equilibrium set of the game.

For the $\mathrm{cHg}$, there exists an interesting principle, the so called Principle of Minimum Differentiation. This principle, coined in [3], comes for the standard interpretation down to that the retailers like to locate together in the middle and formally that $E=\{(L / 2, L / 2)\}$. Note that in the discrete case there only is a middle if $m$ is even. It is reasonable to formalize this principle as follows:

Definition 3.1. a. In the continuous case the Principle of Minimum Differentiation holds if $E=\{(L / 2, L / 2)\}$. b. In the discrete case the Principle of Minimum Differentiation holds if $E=(p, p)$ if $m=2 p$ and $\emptyset \neq E \subseteq$ $\{(p, p),(p+1, p+1),(p, \overline{p+1),(p+1, p)\} \text { if } m=2 p+1 . \diamond}$

The player symmetry implies that ${ }^{4}$ the best-response correspondences $B_{1}, B_{2}: X \multimap X$ are identical:

$$
B_{1}=B_{2}=: B \text {. }
$$

The location symmetry implies that for every $x \in X$

$$
B(v-x)=\{v\}-B(x) .
$$

Player symmetry also implies for every $\left(e_{1}, e_{2}\right) \in E$ that $\left\{\left(e_{1}, e_{2}\right),\left(e_{2}, e_{1}\right)\right\} \subseteq E$ and location symmetry that $\left\{\left(e_{1}, e_{2}\right),\left(v-e_{1}, v-e_{2}\right)\right\} \subseteq E$. Thus

$$
\left(e_{1}, e_{2}\right) \in E \Rightarrow\left\{\left(e_{1}, e_{2}\right),\left(e_{2}, e_{1}\right),\left(v-e_{1}, v-e_{2}\right),\left(v-e_{2}, v-e_{1}\right)\right\} \subseteq E .
$$

This observation makes that we like to see $\left(e_{1}, e_{2}\right),\left(e_{2}, e_{1}\right),\left(v-e_{1}, v-e_{2}\right),\left(v-e_{2}, v-e_{1}\right)$ as the same equilibrium. We formalize this by defining on $E$ the relation $\sim$ by

$$
\left(e_{1}, e_{2}\right) \sim\left(e_{1}^{\prime}, e_{2}^{\prime}\right) \text { means: }\left(e_{1}, e_{2}\right) \in\left\{\left(e_{1}^{\prime}, e_{2}^{\prime}\right),\left(e_{2}^{\prime}, e_{1}^{\prime}\right),\left(v-e_{1}^{\prime}, v-e_{2}^{\prime}\right),\left(v-e_{2}^{\prime}, v-e_{1}^{\prime}\right)\right\} .
$$

It is straightforward to check that this relation is an equivalence relation. Denote by $[E]$, the set of its equivalence classes, to be called equilibrium classes, and by $\left[\left(e_{1}, e_{2}\right)\right]$ the equilibrium class of $\left(e_{1}, e_{2}\right) \in E$. We have

$$
\left[\left(e_{1}, e_{2}\right)\right]=\left\{\left(e_{1}, e_{2}\right),\left(e_{2}, e_{1}\right),\left(v-e_{1}, v-e_{2}\right),\left(v-e_{2}, v-e_{1}\right)\right\}
$$

By the multiplicity of an equilibrium we understand the number of elements of its equilibrium class. Of course, if the game has a unique equilibrium, then there is just one equilibrium class consisting of this equilibrium and this equilibrium has multiplicity 1 . Note that with the action distance of an action profile $\left(x_{1}, x_{2}\right)$ defined by $\left|x_{2}-x_{1}\right|$, each element of a given equilibrium class has the same action distance. Also note that (4) implies:

$$
\# E=1 \Leftrightarrow E=\left\{\left(\frac{v}{2}, \frac{v}{2}\right)\right\}
$$

Thus: if there is a unique equilibrium in the discrete case, then $v$ is even. And: if there is a unique equilibrium, then the Principle of Minimum Differentiation holds.

The following proposition is easy to prove: ${ }^{5}$

Proposition 3.2. Each equilibrium has multiplicity 1, 2 or 4 . Even: for every $\left(e_{1}, e_{2}\right) \in E$

$$
\begin{gathered}
\#\left[\left(e_{1}, e_{2}\right)\right]=1 \Leftrightarrow e_{1}=e_{2} \wedge e_{1}+e_{2}=v ; \\
\#\left[\left(e_{1}, e_{2}\right)\right]=2 \Leftrightarrow\left[e_{1}=e_{2} \wedge e_{1}+e_{2} \neq v\right] \vee\left[e_{1} \neq e_{2} \wedge e_{1}+e_{2}=v\right] ; \\
\#\left[\left(e_{1}, e_{2}\right)\right]=4 \Leftrightarrow\left[e_{1} \neq e_{2} \wedge e_{1}+e_{2} \neq v\right] . \diamond
\end{gathered}
$$

\footnotetext{
${ }^{4}$ We denote a correspondence with the symbol $\multimap$.

${ }^{5}$ Note that the three bi-implications in this statement imply that $\#\left[\left(e_{1}, e_{2}\right)\right] \neq 3$.
} 


\section{Potentials}

\subsection{Potential games}

Consider a game in strategic form. Denote its player set by $N:=\{1, \ldots, n\}$, the strategy set of player $i$ by $X_{i}$ and his payoff function by $u_{i}$. We denote $\mathbf{X}:=X_{1} \times \cdots \times X_{n}, \mathbf{X}_{\hat{\imath}}:=X_{1} \times \cdots \times X_{i-1} \times X_{i+1} \times \cdots \times X_{n}$, identify $\mathbf{X}$ with $X_{i} \times \mathbf{X}_{\hat{\imath}}$, and accordingly write $\mathbf{x} \in \mathbf{X}$ as $\mathbf{x}=\left(x_{i} ; \mathbf{x}_{\hat{\imath}}\right)$.

A function $P: X_{1} \times \cdots \times X_{n} \rightarrow \mathbb{R}$ is called a

- exact potential ( [11] ) if for all $i \in N, \mathbf{z} \in \mathbf{X}_{\hat{\imath}}$ and $a_{i}, b_{i} \in X_{i}$

$$
u_{i}\left(a_{i} ; \mathbf{z}\right)-u_{i}\left(b_{i} ; \mathbf{z}\right)=P\left(a_{i} ; \mathbf{z}\right)-P\left(b_{i} ; \mathbf{z}\right) .
$$

- generalised ordinal potential ( [11] ) if for all $i \in N, \mathbf{z} \in \mathbf{X}_{\hat{\imath}}$ and $a_{i}, b_{i} \in X_{i}$

$$
u_{i}\left(a_{i} ; \mathbf{z}\right)<u_{i}\left(b_{i} ; \mathbf{z}\right) \Rightarrow P\left(a_{i} ; \mathbf{z}\right)<P\left(b_{i} ; \mathbf{z}\right) .
$$

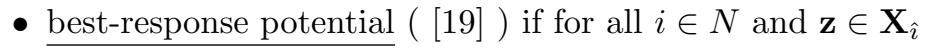

$$
B_{i}(\mathbf{z})=\operatorname{argmax}_{x_{i} \in X_{i}} P\left(x_{i} ; \mathbf{z}\right) .
$$

- quasi potential ( [15] ) if

$$
E=\operatorname{argmax} P .
$$

\subsection{The $\mathbf{d H g}$ is a best-response potential game}

In this subsection we show that the $\mathrm{dHg}$ is a best-response potential game in the sense of [19]. ${ }^{6}$ This means that there exists a best-response potential, i.e. a function $P: S \times S \rightarrow \mathbb{R}$ such that

$$
B_{1}\left(x_{2}\right)=\operatorname{argmax}_{x_{1} \in S} P\left(x_{1}, x_{2}\right)\left(x_{2} \in S\right), \quad B_{2}\left(x_{1}\right)=\operatorname{argmax}_{x_{2} \in S} P\left(x_{1}, x_{2}\right)\left(x_{1} \in S\right) .
$$

As (3) holds and the next theorem deals with a symmetric best-response potential $P^{\bullet}$, we have, with the correspondence $B^{\bullet}: S \multimap S$ defined by

$$
B^{\bullet}\left(x_{2}\right):=\arg \max _{x_{1} \in S} P^{\bullet}\left(x_{1}, x_{2}\right)
$$

there to show that

$$
B=B^{\bullet}
$$

Define $\hat{P}: S \times S \rightarrow \mathbb{R}$ by

$$
\hat{P}\left(x_{1}, x_{2}\right):= \begin{cases}L\left(\min \left\{x_{1}, x_{2}\right\}\right)+R\left(\max \left\{x_{1}, x_{2}\right\}\right)+\frac{1}{2} M\left(x_{1}, x_{2}\right) & \text { if } x_{1} \neq x_{2}, \\ L\left(x_{1}\right)+R\left(x_{1}\right)-f(0) & \text { if } x_{1}=x_{2}\end{cases}
$$

Of course $\hat{P}\left(x_{1}, x_{2}\right)=\hat{P}\left(x_{2}, x_{1}\right)$ holds. Noting the formulas $L(m-a)=R(a)$ and $M(m-a, m-b)=M(a, b)$, we also see that $\hat{P}\left(m-x_{1}, m-x_{2}\right)=\hat{P}\left(x_{1}, x_{2}\right)$.

Theorem 4.1. The discrete Hotelling game is a best-response potential game. Moreover,

${ }^{6}$ For potential games we refer to $[11,13]$ and references therein. 
(1) if $f$ is strictly decreasing, then $P^{\bullet}: S \times S \rightarrow \mathbb{R}$ defined by

$$
P^{\bullet}\left(x_{1}, x_{2}\right):= \begin{cases}\hat{P}\left(x_{1}, x_{2}\right) & \text { if }\left(x_{1}, x_{2}\right) \neq\left(\frac{m}{2}, \frac{m}{2}\right), \\ \hat{P}\left(x_{1}, x_{2}\right)+\frac{1}{2} f(0) & \text { if }\left(x_{1}, x_{2}\right)=\left(\frac{m}{2}, \frac{m}{2}\right)\end{cases}
$$

is a best-response potential.

(2) if $f$ is constant, say $f=c$, then $P^{\bullet}: S \times S \rightarrow \mathbb{R}$ defined by

$$
P^{\bullet}\left(x_{1}, x_{2}\right):=\hat{P}\left(x_{1}, x_{2}\right)-r \min \left\{\left|\frac{m}{2}-x_{1}\right|,\left|\frac{m}{2}-x_{2}\right|\right\},
$$

where $r$ is any constant such that $0<r<\frac{c}{2}$, is a best-response potential. $\diamond$

Proof. We have to show that (5) holds. As $P^{\bullet}\left(x_{1}, x_{2}\right)=P^{\bullet}\left(m-x_{1}, m-x_{2}\right)$, it suffices to show $B\left(x_{2}\right)=B^{\bullet}\left(x_{2}\right)$ for $x_{2} \leq \frac{m}{2}$.

(1). We distinguish two cases.

Case $x_{2}<\frac{m}{2}$ : first, if $x_{1}<x_{2}$, then for $x_{1}^{\prime}=2 x_{2}-x_{1}, M\left(x_{1}, x_{2}\right)=M\left(x_{2}, x_{1}^{\prime}\right)$. Noting that $P^{\bullet}\left(x_{1}, x_{2}\right)=$ $\hat{P}\left(x_{1}, x_{2}\right)$ for any $x_{1} \in S$,

$$
\begin{gathered}
u_{1}\left(x_{1}^{\prime}, x_{2}\right)-u_{1}\left(x_{1}, x_{2}\right)=R\left(x_{1}^{\prime}\right)-L\left(x_{1}\right) \\
>P^{\bullet}\left(x_{1}^{\prime}, x_{2}\right)-P^{\bullet}\left(x_{1}, x_{2}\right)=\left(L\left(x_{2}\right)+R\left(x_{1}^{\prime}\right)\right)-\left(L\left(x_{1}\right)+R\left(x_{2}\right)\right) \\
=\left(\sum_{z=0}^{x_{2}} f(z)-\sum_{z=0}^{x_{1}} f(z)\right)+\left(\sum_{z=0}^{m-x_{1}^{\prime}} f(z)-\sum_{z=0}^{m-x_{2}} f(z)\right) \\
=\sum_{z=x_{1}+1}^{x_{2}} f(z)-\sum_{z=m-x_{1}^{\prime}+1}^{m-x_{2}} f(z)>0,
\end{gathered}
$$

where the first inequality is obtained from $L\left(x_{2}\right)<R\left(x_{2}\right)$, and the last by $x_{1}+1<m-x_{1}^{\prime}+1$ and $x_{2}-$ $\left(x_{1}+1\right)=\left(m-x_{2}\right)-\left(m-x_{1}^{\prime}+1\right)$. Hence $x_{1} \geq x_{2}$ if $x_{1} \in B\left(x_{2}\right) \cup B^{\bullet}\left(x_{2}\right)$. Second, if $x_{1}=x_{2}$, then $M\left(x_{2}, x_{1}\right)=M\left(x_{2}, x_{1}+1\right)=0$, and

$$
\begin{aligned}
2\left(u_{1}\left(x_{1}+1, x_{2}\right)-u_{1}\left(x_{1}, x_{2}\right)\right) & =2 R\left(x_{2}+1\right)-\left(L\left(x_{2}\right)+R\left(x_{2}\right)-f(0)\right) \\
\geq P^{\bullet}\left(x_{1}+1, x_{2}\right)-P^{\bullet}\left(x_{1}, x_{2}\right) & =L\left(x_{2}\right)+R\left(x_{2}+1\right)-\left(L\left(x_{2}\right)+R\left(x_{2}\right)+f(0)\right) \\
=\sum_{z=0}^{m-x_{2}-1} f(z)-\sum_{z=0}^{m-x_{2}} f(z)+f(0) & =-f\left(m-x_{1}\right)+f(0)>0,
\end{aligned}
$$

where the first weak inequality is by $R\left(x_{2}+1\right)-L\left(x_{2}\right)=\sum_{z=0}^{m-x_{2}-1} f(z)-\sum_{z=0}^{x_{2}} f(z) \geq 0$ due to $m-x_{2}-1 \geq x_{2}$. Hence $x_{1}>x_{2}$ if $x_{1} \in B\left(x_{2}\right) \cup B^{\bullet}\left(x_{2}\right)$. Then $B\left(x_{2}\right)$ equals the set of maximizers of $u_{1}\left(\cdot, x_{2}\right)=R(\cdot)+\frac{1}{2} M\left(\cdot, x_{2}\right)$ over $x_{1}>x_{2}$, and $B^{\bullet}\left(x_{2}\right)$ equals the set of maximizers of $P^{\bullet}\left(\cdot, x_{2}\right)=L\left(x_{2}\right)+R(\cdot)+\frac{1}{2} M\left(\cdot, x_{2}\right)$ over $x_{1}>x_{2}$. Since $L\left(x_{2}\right)$ is a constant, $B\left(x_{2}\right)=B^{\bullet}\left(x_{2}\right)$.

Case $x_{2}=\frac{m}{2}$ : in this case $B\left(x_{2}\right)$ and $B^{\bullet}\left(x_{2}\right)$ are symmetric in that $y \in B\left(x_{2}\right) \Leftrightarrow m-y \in B\left(x_{2}\right)$ and $y \in B^{\bullet}\left(x_{2}\right) \Leftrightarrow m-y \in B^{\bullet}\left(x_{2}\right)$. Hence $B\left(x_{2}\right) \cap\left[x_{2}, m\right] \neq \emptyset$ and $B^{\bullet}\left(x_{2}\right) \cap\left[x_{2}, m\right] \neq \emptyset$. We are done if $B\left(x_{2}\right) \cap\left[x_{2}, m\right]=B^{\bullet}\left(x_{2}\right) \cap\left[x_{2}, m\right]$ is shown. Now, observe:

$$
u_{1}\left(x_{1}, x_{2}\right)=\left\{\begin{array}{l}
R\left(x_{1}\right)-\frac{1}{2} f(0) \text { and } P^{\bullet}\left(x_{1}, x_{2}\right)=L\left(x_{2}\right)+R\left(x_{1}\right)-\frac{1}{2} f(0) \text { if } x_{1}=x_{2}, \\
R\left(x_{1}\right)+\frac{1}{2} M\left(x_{1}, x_{2}\right) \text { and } P^{\bullet}\left(x_{1}, x_{2}\right)=L\left(x_{2}\right)+R\left(x_{1}\right)+\frac{1}{2} M\left(x_{1}, x_{2}\right) \text { if } x_{1}>x_{2} .
\end{array}\right.
$$

Thus $P^{\bullet}\left(x_{1}, x_{2}\right)=u_{1}\left(x_{1}, x_{2}\right)+L\left(x_{2}\right)$ for all $x_{1} \geq x_{2}$, and $\arg \max _{x_{1} \geq x_{2}} P^{\bullet}\left(x_{1}, x_{2}\right)=\arg \max _{x_{1} \geq x_{2}} u_{1}\left(x_{1}, x_{2}\right)$ since $L\left(x_{2}\right)$ is constant. Hence $B\left(x_{1}, x_{2}\right) \cap\left[x_{2}, m\right]=B^{\bullet}\left(x_{1}, x_{2}\right) \cap\left[x_{2}, m\right]$. 
(2). Note that $G$ is $c(m+1)$-sum and

$$
\hat{P}\left(x_{1}, x_{2}\right)= \begin{cases}c\left(m+1-\frac{1}{2}\left(\left|x_{2}-x_{1}\right|-1\right)\right) & \text { if } x_{1} \neq x_{2} \\ c(m+1) & \text { if } x_{1}=x_{2}\end{cases}
$$

We distinguish three cases.

Case $x_{2} \leq \frac{m}{2}-1$ : here we can verify that $B\left(x_{2}\right)=\left\{x_{2}+1\right\}$. Notice that $\hat{P}\left(x_{2}, x_{2}\right)=\hat{P}\left(x_{2}+1, x_{2}\right)$ and $r \min \left\{\frac{m}{2}-x_{2}, \frac{m}{2}-x_{2}\right\}>r \min \left\{\frac{m}{2}-\left(x_{2}+1\right), \frac{m}{2}-x_{2}\right\}$, i.e., $P^{\bullet}\left(x_{2}, x_{2}\right)<P^{\bullet}\left(x_{2}+1, x_{2}\right) ;$ and $\hat{P}\left(x_{1}, x_{2}\right) \leq \hat{P}\left(x_{2}+\right.$ $\left.1, x_{2}\right)$ and $r \min \left\{\frac{m}{2}-x_{1}, \frac{m}{2}-x_{2}\right\}>r \min \left\{\frac{m}{2}-\left(x_{2}+1\right), \frac{m}{2}-x_{2}\right\}$ for all $x_{1}<x_{2}$, i.e., $P^{\bullet}\left(x_{1}, x_{2}\right)<P^{\bullet}\left(x_{2}+1, x_{2}\right)$ for all $x_{1}<x_{2}+1$.

Also,

$$
P^{\bullet}\left(x_{1}, x_{2}\right)<P^{\bullet}\left(x_{2}+1, x_{2}\right) \text { for all } x_{1}>x_{2}+1 \text {. }
$$

To see this, note that $\hat{P}\left(x_{1}, x_{2}\right)-\hat{P}\left(x_{2}+1, x_{2}\right)=-\frac{c}{2}\left(x_{1}-x_{2}-1\right)$ for all $x_{1}>x_{2}+1$, and $r \min \left\{\left|\frac{m}{2}-x_{1}\right|, \frac{m}{2}-\right.$ $\left.x_{2}\right\}-r \min \left\{\frac{m}{2}-\left(x_{2}+1\right), \frac{m}{2}-x_{2}\right\}=-r\left(x_{1}-x_{2}-1\right)$ if $x_{1} \leq m-x_{2}$, and zero if $x_{1}>m-x_{2}$. In either case their sum $\hat{P}\left(x_{1}, x_{2}\right)-\hat{P}\left(x_{2}+1, x_{2}\right)$ is negative (note that $\left.-\frac{c}{2}+r<0\right)$. Hence $B^{\bullet}\left(x_{2}\right)=B\left(x_{2}\right)=\left\{x_{2}+1\right\}$ if $x_{2} \leq \frac{m}{2}-1$.

Case $\frac{m}{2}-1<x_{2}<\frac{m}{2}$ : now $m$ is odd and $x_{2}=\frac{m-1}{2}$. We can see that

$$
B\left(x_{2}\right)=\left\{x_{2}, x_{2}+1\right\}
$$

Since $\hat{P}\left(x_{2}, x_{2}\right)=\hat{P}\left(x_{2}+1, x_{2}\right)>\hat{P}\left(x_{1}, x_{2}\right)$ for all $x_{1} \notin\left\{x_{2}, x_{2}+1\right\}$ and $r \min \left\{\frac{m}{2}-x_{2}, \frac{m}{2}-x_{2}\right\}=r \min \left\{\frac{m}{2}-\right.$ $\left.\left(x_{2}+1\right), \frac{m}{2}-x_{2}\right\}=r \min \left\{\left|\frac{m}{2}-x_{1}\right|, \frac{m}{2}-x_{2}\right\}$ for all $x_{1} \notin\left\{x_{2}, x_{2}+1\right\}$, we have $B^{\bullet}\left(x_{2}\right)=B\left(x_{2}\right)=\left\{x_{2}, x_{2}+1\right\}$ if $\frac{m}{2}-1<x_{2}<\frac{m}{2}$.

Case $x_{2}=\frac{m}{2}$ : in this case we can see that $B\left(x_{2}\right)=\left\{x_{2}\right\}$. Since $\hat{P}\left(x_{2}, x_{2}\right)>\hat{P}\left(x_{1}, x_{2}\right)$ and $r \min \left\{\frac{m}{2}-x_{2}, \frac{m}{2}-\right.$ $\left.x_{2}\right\}=r \min \left\{\left|\frac{m}{2}-x_{1}\right|, \frac{m}{2}-x_{2}\right\}$ for all $x_{1} \neq x_{2}$, we have $B^{\bullet}\left(x_{2}\right)=B\left(x_{2}\right)=\left\{x_{2}\right\}$ if $x_{2}=\frac{m}{2}$.

\subsection{Other potentials}

Having Theorem 4.1, the question may arise whether a $\mathrm{dHg}$ admits another type of potential, like an exact potential or a generalized ordinal potential game.

If $m=1$, then $u_{1}=u_{2}$, i.e. the game is an identical interest game and therefore an exact potential game. The following proposition shows that our class of $\mathrm{dHg}$ is neither contained in the class of exact potential games nor in the class of generalized ordinal potential games.

Theorem 4.2. Consider the $d H g$ with $f(d)=w^{d}$ and $m=3$.

(1) The game is for no $w$ an exact potential game.

(2) For $w>\frac{1}{2}$ the game is not a generalized ordinal potential game. $\diamond$

Proof. Consider the cyclic path of length 4

$$
\gamma:=\left(\mathbf{x}^{(1)}, \mathbf{x}^{(2)}, \mathbf{x}^{(3)}, \mathbf{x}^{(4)}, \mathbf{x}^{(5)}\right)=((2,0),(1,0),(1,3),(2,3),(2,0)) .
$$

This cyclic path is simple, i.e. $\mathbf{x}^{(r)} \neq \mathbf{x}^{(s)}(1 \leq r<s \leq 4)$.

(1). Using the bi-matrix (2), we obtain

$$
\begin{array}{ccc} 
& \left(u_{1}\left(\mathbf{x}^{(2)}\right)-u_{1}\left(\mathbf{x}^{(1)}\right)\right)+\left(u_{2}\left(\mathbf{x}^{(3)}\right)-u_{2}\left(\mathbf{x}^{(2)}\right)\right)+\left(u_{1}\left(\mathbf{x}^{(4)}\right)-u_{1}\left(\mathbf{x}^{(3)}\right)\right)+\left(u_{2}\left(\mathbf{x}^{(5)}\right)-u_{2}\left(\mathbf{x}^{(4)}\right)\right) \\
= & \left(u_{1}(1,0)-u_{1}(2,0)\right)+\left(u_{1}(3,1)-u_{1}(0,1)\right)+\left(u_{1}(2,3)-u_{1}(1,3)\right)+\left(u_{1}(0,2)-u_{1}(3,2)\right) \\
= & \left(1+w+w^{2}\right)-\left(1+w+\frac{w}{2}\right)+\left(1+\frac{w}{2}\right)-1+\left(1+w+w^{2}\right)-\left(1+w+\frac{w}{2}\right)+\left(1+\frac{w}{2}\right)-1 \\
= & 2 w^{2} \neq 0 .
\end{array}
$$


As explained in [11], this calculation implies that the game is not an exact potential game.

(2). By definition $\gamma$ is an improvement path if and only if $u_{1}(2,0)<u_{1}(1,0) \wedge u_{2}(1,0)<u_{2}(1,3) \wedge u_{1}(1,3)<$ $u_{1}(2,3) \wedge u_{2}(2,3)<u_{2}(2,0)$. So $\gamma$ is an improvement path

$$
\begin{gathered}
\Leftrightarrow u_{1}(2,0)<u_{1}(1,0) \wedge u_{1}(0,1)<u_{1}(3,1) \wedge u_{1}(1,3)<u_{1}(2,3) \wedge u_{1}(3,2)<u_{1}(0,2) \\
\Leftrightarrow\left[\frac{w}{2}<w^{2} \wedge 0<\frac{w}{2} \wedge \frac{w}{2}<w^{2} \wedge 0<\frac{w}{2}\right] \Leftrightarrow \frac{1}{2}<w .
\end{gathered}
$$

If the game has a generalized ordinal potential then it cannot have a non-trivial cyclic improvement path ( [11]). Thus for $w>1 / 2$ the game is not a generalized ordinal potential game.

\section{Structure of the Equilibrium Set in CASE $f(d)=w^{d}$}

We have seen that the $\mathrm{dHg}$ has an equilibrium. However, one may wish to have more insight in the structure of its equilibrium set $E$.

Below we present for the case $f(d)=w^{d}$, where $0<w \leq 1$, some results concerning the structure of $E$. These results will be proved by a straightforward inspection of the explicit formulas in Theorems 1 and 2 in [18]. Obtaining these formulas (for $w \neq 1$ ) was quite complicated: 10 cases were distinguished.

The analysis of the case of inelastic demand, meaning that $w=1$, is simple: for the best-response correspondence $B=B_{1}=B_{2}$ we have

$$
\begin{gathered}
B(x)=\left\{\begin{array}{l}
\{x+1\} \text { if } x<p, \\
\{x\} \text { if } x=p, \\
\{x-1\} \text { if } x>p
\end{array} \quad \text { if } m\right. \text { is even, } \\
B(x)=\left\{\begin{array}{l}
\{x+1\} \text { if } x<p, \\
\{x, x+1\} \text { if } x=p, \\
\{x-1, x\} \text { if } x=p+1, \\
\{x-1\} \text { if } x>p+1,
\end{array} \text { if } m\right. \text { is odd }
\end{gathered}
$$

This implies for $w=1$ :

$$
E=\left\{\begin{array}{l}
\{(p, p)\} \text { if } m \text { is even, } \\
\{(p, p),(p+1, p+1),(p, p+1),(p+1, p)\} \text { if } m \text { is odd }
\end{array}\right.
$$

So for $w=1$ the Principle of Minimum Differentiation holds. Concerning equilibrium classes for $w=1$, note that if $m$ is even, then $[E]=\{\{(p, p)\}\}$ and if $m$ is odd, then $[E]=\{\{(p, p),(p+1, p+1)\},\{(p, p+1),(p+1, p)\}\}$.

As Propositions 5.1 and 5.2 make crystally clear, the following value of the distance factor plays an important role: ${ }^{7}$

$$
w_{c}:=\left\{\begin{array}{l}
2^{-1 / p} \text { if } m \text { is even, } \\
2^{-1 /(p-1)} \text { if } m \text { is odd. }
\end{array}\right.
$$

Proposition 5.1. (1) Suppose $m$ is even.

(a) If $w>w_{c}$, then $E=\{(p, p)\}$.

(b) If $w=w_{c}$, then $E=\{(p, p),(p-1, p),(p, p-1),(p+1, p),(p, p+1)\}$.

(c) If $w<w_{c}$, then the action distance of each equilibrium is at least 1.

(2) Suppose $m$ is odd with $w \neq 1$.

(a) If $w>w_{c}$, then $E=\{(p, p+1),(p+1, p)\}$.

(b) If $w=w_{c}$, then $E=\{(p-1, p+1),(p+1, p-1),(p+2, p),(p, p+2),(p+2, p-1)$, $(p-1, p+2),(p, p+1),(p+1, p)\}$.

(c) If $w<w_{c}$, then the action distance of each equilibrium is at least $3 . \diamond$

${ }^{7}$ Here $2^{-1 /(p-1)}$ with $p=1$ or $p=0$ has to be interpreted as 0. 
Proof. (1a). By Theorems 1 and 2(Ie) in [18].

(1b). By Theorems 1 and 2(Id) in [18].

(1c). By Theorems 1 and 2(I) in [18].

(2a), (2b). By Theorems 1 and 2(IId) in [18].

(2c). By Theorems 1 and 2(II) in [18].

Proposition 5.1(1) implies that for $m$ even: there exists a symmetric equilibrium if and only if $w \geq w_{c}$. With Proposition 5.1(2) we see that for $m$ odd: there exists a symmetric equilibrium if and only if $w=1$.

Proposition 5.2. The Principle of Minimum Differentiation holds if and only if $w>w_{c} . \diamond$

Proof. By Proposition 5.1 and (8), this is clear if $m$ is odd, or if $m$ is even and $w \geq w_{c}$. Now suppose $m$ is even and $w<w_{c}$. Since $m$ is even, by definition, the Principle holds if and only if $E=\{(p, p)\}$. But by $w<w_{c}$ the action distance is at least 1 , so $E \neq\{(p, p)\}$; the Principle does not hold.

The inequality $w>w_{c}$ may be interpreted as " $f$ is "sufficiently inelastic". Note that $w>2^{-1 / p} \Leftrightarrow f(p)>$ $\frac{1}{2} f(0)$ and $w>2^{-1 /(p-1)} \Leftrightarrow f(p-1)>\frac{1}{2} f(0)$.

Now let us consider the equilibrium classes. First suppose $m$ is even. Then if $w>w_{c}$, Proposition 5.1(1) shows that there is 1 equilibrium class and if $w=w_{c}$, there are 2 equilibrium classes. For $w<w_{c}$ the situation is more complicated. Next suppose $m$ is odd. Then, if $w_{c}<w<1$, Proposition $5.1(2)$ shows that there is 1 equilibrium class and if $w=w_{c}$ there are three equilibrium classes. Again, if $w<w_{c}$, the situation is more complicated. However, Proposition 5.3(4) holds.

Proposition 5.3. (1) The game has a unique equilibrium if and only if $m$ is even and $w=1$.

(2) If $\left(e_{1}, e_{2}\right) \in E$

(a) and $m$ is even, then $e_{1}+e_{2} \in\{m-1, m, m+1\}$;

(b) and $m$ is odd then $e_{1}+e_{2} \in\{m-2, m-1, m, m+1, m+2\}$.

(3) $1 \leq \# E \leq 8$.

(4) There are at most 3 equilibrium classes. $\diamond$

Proof. By Theorems 1 and 2 in [18].

An example with 3 equilibrium classes is (as follows from Theorems 1 and 2(II) in [18]): $m=11$ and $w=2^{-1 / 2}$. Then $(p=5$ and $)[E]=\{\{(p-2, p+2),(p+2, p-2),(p+3, p-1),(p-1, p+3)\},\{(p-1, p+$ $2),(p+2, p-1)\},\{(p-2, p+3),(p+3, p-2)\}\}$.

A question for further research: what remains of these results for a general $\mathrm{dHg}$ ?

\section{A CONJECTURE}

Various classes of finite games in strategic form that have a (pure) equilibrium are identified. We mention here: potential games, supermodular games, symmetric games with integrally concave payoffs ( [9]) and games with increasing best-response correspondences.

In [18] it has been shown that a $\mathrm{dHg}$ may not be a supermodular game and that a $\mathrm{dHg}$ may not have integrally concave payoffs. In the present article we have shown that the $\mathrm{dHg}$ is a best-response potential game. Below we look to increasing best-response correspondences in case of the demand function $f(d)=w^{d}$.

So consider the best-response correspondence $B=B_{1}=B_{2}: S \multimap S$. For $w=1$ it is from (6) and (7) easy to check that $B$ has an increasing selection. However this does not hold for all $m$ and $w$ : indeed, the next proposition implies that for $m$ odd and $w \neq 1$ it holds that $B(p) \subseteq\{p+1, \ldots, m\}$ and $B(p+1) \subseteq\{0, \ldots, p\}$.

Proposition 6.1. Suppose $w \neq 1$ or $m$ is even.

(1) If $x>m / 2$, then $B(x) \subseteq\{0,1, \ldots, x-1\}$.

(2) If $x<m / 2$, then $B(x) \subseteq\{x+1, x+2, \ldots, m\}$.

Proof. By Proposition 3 in [18]. 
In particular $B$ does not have an increasing selection in case $m=7$ and $w=1 / 2$. Then the bi-matrix game is

$$
\left(\begin{array}{cccccccc}
\left(\frac{255}{256}, \frac{255}{256}\right) & \left(1, \frac{127}{64}\right) & \left(\frac{5}{4}, \frac{71}{32}\right) & \left(\frac{3}{2}, \frac{39}{16}\right) & \left(\frac{13}{8}, \frac{5}{2}\right) & \left(\frac{7}{4}, \frac{5}{2}\right) & \left(\frac{29}{16}, \frac{37}{16}\right) & \left(\frac{15}{8}, \frac{15}{8}\right) \\
\left(\frac{127}{64}, 1\right) & \left(\frac{159}{128}, \frac{159}{128}\right) & \left(\frac{3}{2}, \frac{63}{32}\right) & \left(\frac{7}{4}, \frac{35}{16}\right) & \left(2, \frac{19}{8}\right) & \left(\frac{17}{8}, \frac{19}{8}\right) & \left(\frac{9}{4}, \frac{9}{4}\right) & \left(\frac{37}{16}, \frac{29}{16}\right) \\
\left(\frac{71}{32}, \frac{5}{4}\right) & \left(\frac{63}{32}, \frac{3}{2}\right) & \left(\frac{87}{64}, \frac{87}{64}\right) & \left(\frac{7}{4}, \frac{31}{16}\right) & \left(2, \frac{17}{8}\right) & \left(\frac{9}{4}, \frac{9}{4}\right) & \left(\frac{19}{8}, \frac{17}{8}\right) & \left(\frac{5}{2}, \frac{7}{4}\right) \\
\left(\frac{39}{16}, \frac{3}{2}\right) & \left(\frac{35}{16}, \frac{7}{4}\right) & \left(\frac{31}{16}, \frac{7}{4}\right) & \left(\frac{45}{32}, \frac{45}{32}\right) & \left(\frac{15}{8}, \frac{15}{8}\right) & \left(\frac{17}{8}, 2\right) & \left(\frac{19}{8}, 2\right) & \left(\frac{5}{2}, \frac{13}{8}\right) \\
\left(\frac{5}{2}, \frac{13}{8}\right) & \left(\frac{19}{8}, 2\right) & \left(\frac{17}{8}, 2\right) & \left(\frac{15}{8}, \frac{15}{8}\right) & \left(\frac{45}{32}, \frac{45}{32}\right) & \left(\frac{31}{16}, \frac{7}{4}\right) & \left(\frac{35}{16}, \frac{7}{4}\right) & \left(\frac{39}{16}, \frac{3}{2}\right) \\
\left(\frac{5}{2}, \frac{7}{4}\right) & \left(\frac{19}{8}, \frac{17}{8}\right) & \left(\frac{9}{4}, \frac{9}{4}\right) & \left(2, \frac{17}{8}\right) & \left(\frac{7}{4}, \frac{31}{16}\right) & \left(\frac{87}{64}, \frac{87}{64}\right) & \left(\frac{63}{32}, \frac{3}{2}\right) & \left(\frac{71}{32}, \frac{5}{4}\right) \\
\left(\frac{37}{16}, \frac{29}{16}\right) & \left(\frac{9}{4}, \frac{9}{4}\right) & \left(\frac{17}{8}, \frac{19}{8}\right) & \left(2, \frac{19}{8}\right) & \left(\frac{7}{4}, \frac{35}{16}\right) & \left(\frac{3}{2}, \frac{63}{32}\right) & \left(\frac{159}{128}, \frac{159}{128}\right) & \left(\frac{127}{64}, 1\right) \\
\left(\frac{15}{8}, \frac{15}{8}\right) & \left(\frac{29}{16}, \frac{37}{16}\right) & \left(\frac{7}{4}, \frac{5}{2}\right) & \left(\frac{13}{8}, \frac{5}{2}\right) & \left(\frac{3}{2}, \frac{39}{16}\right) & \left(\frac{5}{4}, \frac{71}{32}\right) & \left(1, \frac{127}{64}\right) & \left(\frac{255}{256}, \frac{255}{256}\right)
\end{array}\right) .
$$

Thus the set $[E]$ of equilibrium classes equals $\{\{(1,4),(4,1),(3,6),(6,3)\},\{(2,5),(5,2)\}\}$ and for the bestresponses we have $B(0)=\{4,5\}, B(1)=\{4,5\}, B(2)=\{5\}, B(3)=\{5,6\}, B(4)=\{1,2\}, B(5)=\{2\}, B(6)=$ $\{2,3\}, B(7)=\{2,3\}$.

As a $\mathrm{dHg}$ is a symmetric game in strategic form with two players we have, letting $B^{2}=B \circ B$,

there exists a Nash equilibrium $\Leftrightarrow B^{2}$ has a fixed point.

And by virtue of Tarski's fixed point, we have

$$
B^{2} \text { has an increasing selection } \Rightarrow B^{2} \text { has a fixed point. }
$$

Thus sufficient for a $\mathrm{dHg}$ to have an equilibrium is that the correspondence $B^{2}$ has an increasing selection.

As in the above example $B^{2}(0)=\{1,2\}, B^{2}(1)=\{1,2\}, B^{2}(2)=\{2\}, B^{2}(3)=\{2,3\}, B^{2}(4)=\{4,5\}, B^{2}(5)=$ $\{5\}, B^{2}(6)=\{5,6\}, B^{2}(7)=\{5,6\}$, we see that in this example $B^{2}$ has an increasing selection. This seems to be true for each $\mathrm{dHg}$ with demand function $f(d)=w^{d}$ :

Conjecture 6.1. If $f(d)=w^{d}$, then the correspondence $B^{2}: S \multimap S$ has an increasing selection. $\diamond$

We have checked this conjecture for many values of $m$ and $w$ with Maple. So also the correctness of the conjecture implies that the discrete Hotelling game with $f(d)=w^{d}$ has a Nash equilibrium. It may be interesting to identify a deeper reason for its validity.

Proposition 6.3 below may be helpful in a further study of the conjecture. It implies that $B$ has an increasing selection on the set $S_{-}:=\left\{x_{2} \in S \mid x_{2}<\frac{m}{2}\right\}$ and on the set $S_{+}:=\left\{x_{2} \in S \mid x_{2}>\frac{m}{2}\right\}$.

For $x_{2} \in S$, the conditional payoff function $u_{1}^{\left(x_{2}\right)}: S \rightarrow \mathbb{R}$ of player 1 is defined by $u_{1}^{\left(x_{2}\right)}\left(x_{1}\right)=u_{1}\left(x_{1}, x_{2}\right)$.

Lemma 6.2. (1) Let $q_{i ; j}:=w^{i}-\frac{1}{2} w^{\left\lfloor\frac{j-i+1}{2}\right\rfloor}$. If $0<x_{1}<x_{2}$, then $8 u_{1}^{\left(x_{2}\right)}\left(x_{1}\right)=q_{x_{1} ; x_{2}}$.

(2) If $m \geq 3$, then $B(x) \subseteq\{1, \ldots, m-1\}$.

(3) Suppose $i, j \in S$ with $0<i<j$.

(a) If $q_{i ; j} \leq 0$, then $q_{k ; j}<0$ for $i<k<j$.

(b) If $q_{i ; j} \geq 0$, then $q_{l ; j}>0$ for $0<l<i$.

(4) Suppose $x_{1}, x_{2} \in S$ with $0<x_{1}<m$, then $x_{1} \in B\left(x_{2}\right) \Rightarrow \Delta u_{1}^{\left(x_{2}\right)}\left(x_{1}+1\right) \leq 0 \leq u_{1}^{\left(x_{2}\right)}\left(x_{1}\right)$.

Proof. For these results, respectively see in [18]: Proposition 2, Proposition 4, Lemma 1 and formula (8).

Proposition 6.3. The functions $\max (B)$ and $\min (B)$ are increasing on $S_{+}$and increasing on $S_{-} . \diamond$

${ }^{8} \Delta u_{1}^{\left(x_{2}\right)}: S \backslash\{0\} \rightarrow \mathbb{R}$ is defined by $\Delta u_{1}^{\left(x_{2}\right)}(x):=u_{1}^{\left(x_{2}\right)}\left(x_{1}\right)-u_{1}^{\left(x_{2}\right)}\left(x_{1}-1\right)$. 
Proof. (6) and (7) show that the statements hold for $w=1$. If $m=1$ or $m=2$, then $\# S_{-}=\# S_{+}=1$ and so the statements are trivial. As, for $x_{2} \in S, B\left(m-x_{2}\right)=\{m\}-B\left(x_{2}\right)$ the correctness of the statements for $S_{-}$ follows from those for $S_{+}$. Now suppose $w \neq 1$ and $m \geq 3$; we prove the statements for $S_{+}$.

First we prove that $\min (B)$ is increasing on $S_{+}$. So fix $x_{2}, x_{2}^{\prime} \in S_{+}$with $x_{2}<x_{2}^{\prime}$ and let $x_{1}=\min B\left(x_{2}\right)$ and $x_{1}^{\prime}=\min B\left(x_{2}^{\prime}\right)$. As $m \geq 3$, we have by Lemma $6.2(2)$

$$
0<x_{1}<m \text { and } 0<x_{1}^{\prime}<m \text {. }
$$

By Lemma 6.2(4), $\Delta u_{1}^{\left(x_{2}\right)}\left(x_{1}\right)=u_{1}^{\left(x_{2}\right)}\left(x_{1}\right)-u_{1}^{\left(x_{2}\right)}\left(x_{1}-1\right) \geq 0$ and $\Delta u_{1}^{\left(x_{2}^{\prime}\right)}\left(x_{1}^{\prime}+1\right) \leq 0$. As $x_{2}>\frac{m}{2}$, Lemma 6.1(1) guarantees

$$
x_{1}<x_{2} .
$$

Since $x_{1}=\min B\left(x_{2}\right)$, we have $u_{1}^{\left(x_{2}\right)}\left(x_{1}\right) \neq u_{1}^{\left(x_{2}\right)}\left(x_{1}-1\right)$ and therefore $\Delta u_{1}^{\left(x_{2}\right)}\left(x_{1}\right)>0$. By Lemma 6.2(1), $q_{x_{1}, x_{2}}>0$. As $0<w<1$ and $x_{2}<x_{2}^{\prime}$, we obtain $0<q_{x_{1}, x_{2}} \leq q_{x_{1}, x_{2}^{\prime}}$. Thus $q_{x_{1}, x_{2}^{\prime}}>0$. By Lemma 6.2(1), $q_{x_{1}^{\prime}+1, x_{2}^{\prime}} \leq$ 0. Lemma 6.2(3) implies $x_{1}^{\prime}+1>x_{1}$ and therefore, as desired, $x_{1}^{\prime} \geq x_{1}$.

With an analogous proof one shows that $\max (B)$ is increasing on $S_{+}$.

\section{Comparing the DHg With the CHG}

The $\mathrm{cHg}$, i.e. the continuous Hotelling game, is the game that we obtain by replacing the formula (1) by

$$
u_{i}\left(x_{1}, x_{2}\right):=\int_{V_{i}\left(x_{1}, x_{2}\right)} f\left(\left|y-x_{i}\right|\right) d y+\int_{V_{0}\left(x_{1}, x_{2}\right)} \frac{f\left(\left|y-x_{i}\right|\right)}{2} d y
$$

where now $S$ is a proper real interval $[0, L]$.

Below we quickly compare results for the $\mathrm{dHG}$ and the $\mathrm{cHg}$. As for the $\mathrm{dHg}$ general results for the structure of the equilibrium set only are available for the demand function $f(d)=w^{d}(0<w \leq 1)$, we do this for this demand function. ${ }^{9}$

A new notion: the function $\sigma: E \rightarrow \mathbb{R}$ defined by $\sigma\left(e_{1}, e_{2}\right)=e_{1}+e_{2}$ is referred to as Nash sum.

The next table compares some of the above results with those in $[8,17]$ :

\begin{tabular}{||c|c|c||}
\hline property/game & $\mathrm{cHg}$ & $\mathrm{dHg}$ \\
\hline \# equilibria & 1,2 & $1,2, \ldots, 8$ \\
\hline multiplicity of equilibrium & 1 or 2 & $1,2,4$ \\
\hline Nash sum & $\mathrm{L}$ & $\mathrm{m}-2, \mathrm{~m}-1, \ldots, \mathrm{m}+2$ \\
\hline \# equilibrium classes & 1 & 1,2 or 3 \\
\hline potential & continuous quasi & best-response \\
\hline
\end{tabular}

More detailed results for the $\mathrm{cHg}$ are contained in [8] and [17]. For example, the $\mathrm{cHg}$ has a continuous best-response potential if and only if $w \leq 2^{-2 / L}$.

A final remark: as $P^{\bullet}$ in Theorem 4.1 is a best-response potential for the $\mathrm{dHg}$, it is well-known that a maximiser of $P^{\bullet}$ is a Nash equilibrium. However it turns out that a Nash equilibrium may not be a maximiser of $P^{\bullet}$, i.e. $P^{\bullet}$ may not be a quasi-potential. That this is true can be checked by an explicit calculation: for the concrete bi-matrix game (with $m=7$ ) in Section 6 the set of maximisers of $P^{\bullet}$ equals $\{(2,5),(5,2)\}$; however, the Nash equilibrium set is larger.

\footnotetext{
${ }^{9}$ For the $\mathrm{cHg}$, in $[8]$ and $[17]$ results can be found for a general (so even not necessarily continuous) $f$.
} 


\section{REFERENCES}

[1] N. Abudaldah, W. Heijman, and P. Heringa. Return of the icecream men. Romanian Journal of Regional Science, 9(2):39-48, 2015.

[2] S. P. Anderson, A. de Palma, and J.-F. Thisse. Discrete Choice Theory of Product Differentiation. MIT Press, Cambridge, 1992.

[3] K. Boulding. Economic Analysis: Microeconomics, volume I. Harper \& Row, New York, 3 edition, 1955.

[4] S. Brusco, M. Dziubínski, and J. Roy. The hotelling-downs model with runoff voting. Games and Economic Behavior, 74:447469, 2012.

[5] C. Dürr and Thang N. K. Nash equilibria in voronoi games on graphs. In Arge L., Hoffmann M., and Welzl E., editors, Algorithms - ESA 2007, volume 4698 of Lecture Notes in Computer Science. Springer, Berlin, Heidelberg, 2007.

[6] H. Hotelling. Stability in competition. The Economic Journal, 39(153):41-57, 1929.

[7] Z. Huang. The mixed strategy equilibrium of the three-firm location game with discrete location choices. Economics Bulletin, 31(3):2109-2116, 2011.

[8] T. Iimura, P. H. M. von Mouche, and T. Watanabe. Best-response potential for hotelling pure location games. Economics Letters, 160:73-77, 2017.

[9] T. Iimura and T. Watanabe. Existence of a pure strategy equilibrium in finite symmetric games where payoff functions are integrally concave. Discrete Applied Mathematics, 166:26-33, 2014.

[10] A. McLennan, P. K. Monteiro, and R. Tourky. Games with discontinuous payoffs; a strengthening of Reny's existence theorem. Econometrica, 79(5):1643-1664, 2011.

[11] D. Monderer and L. Shapley. Potential games. Games and Economic Behavior, 14:124-143, 1996.

[12] M. Núñez and M. Scarsini. Competing over a finite number of locations. Economic Theory Bulletin, 4:125-136, 2016.

[13] J. Park. Potential games with incomplete preferences. Journal of Mathematical Economics, 61:58-66, 2015.

[14] E. Prisner. Best response digraphs for two location games on graphs. In L. A. Petrosjan and N. A. Zenkevich, editors, Contributions to Game Theory and Management, volume IV, pages 378-388. Saint Petersburg, 2011.

[15] B. C. Schipper. Pseudo-potential games. Technical report, University of Bonn, Bonn, Germany, 2004.

[16] B. H. Stevens. An application of game theory to a problem in location strategy. Papers and Proceedings of the Regional Science Association, 7:143-157, 1961.

[17] P. H. M. von Mouche. The continuous Hotelling pure location game with elastic demand revisited. volume 12095 of Motor 2020, Lecture Notes in Computer Science, pages 246-262. Springer Nature Switzerland AG, 2020.

[18] P. H. M. von Mouche and W. Pijnappel. The Hotelling bi-matrix game. Optimization Letters, 12(1):187-202, 2018.

[19] M. Voorneveld. Best-response potential games. Economics Letters, 66:289-295, 2000. 